\title{
MAGNETIC FLUX LOSS FROM INTERSTELLAR CLOUDS WITH VARIOUS GRAIN SIZE DISTRIBUTIONS
}

\author{
Ryoichi Nishi and Takenori Nakano \\ Department of Physics, Kyoto University, Kyoto, Japan \\ Toyoharu Umebayashi \\ Data Processing Center, Yamagata University, Yamagata, Japan
}

A cloud with magnetic flux greater than some critical value cannot contract dynamically. The magnetic flux to mass ratio for a cloud is several hundred to $10^{5}$ times greater than that for a magnetic star. Thus a cloud with supercritical flux must lose some magnetic flux before dynamical contraction begins, and a cloud must lose most of its initial flux at some stage of star formation. The magnetic field is dissipated by the ambipolar diffusion or plasma drift and by the ohmic dissipation. The efficiencies of these processes depend on the densities of various charged particles, for which the reactions on grain surface are important. The previous studies on the field dissipation were based on the assumption that all grains have the same radius $a \simeq 0.1 \mu \mathrm{m}$ (Nakano \& Umebayashi 1980, 1986; Nakano 1984). However, the interstellar grains have a wide size distribution like the MRN distribution $\left(d n_{g r} / d a=A n_{H} a^{-3.5}\right.$ for $\left.50 \AA<a<2500 \AA\right)$. In addition recent observations suggest the existence of very small "grains" ( $a$ as small as $3 \AA$ ). Small grains have two effects on the field dissipation:

(1) Because of the large total surface of small grains, recombination of ions and electrons on them is efficient and suppresses the ion density. This has an effect of enhancing the field dissipation.

(2) Grains are usually charged, and a smaller charged grain couples more strongly with magnetic field. This has an effect of suppressing the field dissipation.

Both effects are large and in opposite directions. We investigate the field dissipation by taking into account the effect of polarization of grains on the collision with ions and electrons for the following four cases of grain size distribution:

(1) The standard MRN distribution $(50 \AA<a<2500 \AA)$;

(2) The MRN distribution with ice-mantle $(a \rightarrow 2.2 a)$;

(3) The extended MRN distribution ( $3 \AA<a<2500 \AA)$;

(4) The standard MRN distribution plus grains with $a=4 \AA\left(2 \times 10^{-7} n_{H}\right)$.

The dissipation time of magnetic field, $t_{B}$, is shorter than the free-fall time $t_{f}$ only at $n_{H}$ higher than $6 \times 10^{11} \mathrm{~cm}^{-3}$ (case 1 ), $3 \times 10^{10} \mathrm{~cm}^{-3}$ (case 2), and $3 \times 10^{13} \mathrm{~cm}^{-3}$ (cases 3 and 4 ), and is more than $10 t_{f}$ at somewhat lower densities. The results for case 1 nearly agree with the previous results. In all cases the magnetic field is strongly coupled to the gas and the magnetic flux of the cloud cannot decrease far below the critical flux at least at $n_{H} \lesssim 3 \times 10^{10} \mathrm{~cm}^{-3}$. 